\title{
SEQUENCES OF INDEPENDENT IDENTICALLY DISTRIBUTED FUNCTIONS IN REARRANGEMENT INVARIANT SPACES
}

\author{
S. V. ASTASHKIN \\ Department of Mathematics and Mechanics, Samara State University \\ Samara, Russia \\ E-mail:astashkn@ssu.samara.ru \\ F. A. SUKOCHEV \\ School of Informatics and Engineering, Flinders University \\ Bedford Park, SA 5042, Australia \\ E-mail: sukochev@infoeng.flinders.edu.au
}

\begin{abstract}
A new set of sufficient conditions under which every sequence of independent identically distributed functions from a rearrangement invariant (r.i.) space on $[0,1]$ spans there a Hilbertian subspace are given. We apply these results to resolve open problems of N. L. Carothers and S. L. Dilworth, and of M. Sh. Braverman, concerning such sequences in concrete r.i. spaces.
\end{abstract}

1. Introduction. Let $X$ be a r.i. space on $[0,1]$, and let $\left\{f_{k}\right\}_{k=1}^{\infty} \subset X$ be a sequence of independent identically distributed random variables (i.i.d.r.v.'s). In this article, we are concerned with the question under which conditions on the space $X$, there exists a constant $C>0$ such that the inequality

$$
C^{-1}\left(\sum_{k=1}^{n} a_{k}^{2}\right)^{1 / 2} \leq\left\|\sum_{k=1}^{n} a_{k} f_{k}\right\|_{X} \leq C\left(\sum_{k=1}^{n} a_{k}^{2}\right)^{1 / 2}
$$

holds for every $n \in \mathbb{N}$ and $\left\{a_{k}\right\}_{k=1}^{n} \subset \mathbb{R}$. It is convenient to recall first some relevant results from [Br, Chapter 3]. Note that only the right inequality in (1.1) is of interest, since the left inequality holds for an arbitrary r.i. $X$ [Br, Lemma 1, p. 52]. The (easy) set of conditions on $X$ and $f_{1}$ necessary for (1.1) to hold is listed in [Br, p. 71] (all unexplained notions from the Banach function space theory are defined in the next section, see also [LT]):

2000 Mathematics Subject Classification: 46A30, 60G50.

Key words and phrases: rearrangement invariant space, i.i.d. sequence.

The paper is in final form and no version of it will be published elsewhere. 
(a) the Köthe bidual $X^{\times \times}$contains the Orlicz space $L_{N}$ generated by the function $N(t)=e^{t^{2}}-1$

(b) $f_{1} \in L_{2}$;

(c) $\mathbb{E} f_{1}:=\int_{0}^{1} f_{1}(x) d x=0$.

To present a set of sufficient conditions for (1.1) to hold, we need first some notations from [Br, Chapter 3]. For a sequence $a=\left(a_{k}\right)_{k=1}^{\infty}$ and a r.v. $f$ on $[0,1]$, we set

$$
Q_{a} f(t)=\sum_{k=1}^{\infty} \lambda\left\{s \in[0,1]:\left|a_{k} f(s)\right|>t\right\}, \quad t>0,
$$

where $\lambda$ is Lebesgue measure. The r.v. $f$ is said to have the property $A_{2}(X)$ (briefly, $\left.f \in A_{2}(X)\right)$ if for all $a \in l_{2}$ the r.i. space $X$ contains all r.v.'s $g$ satisfying the condition $\lambda\{s \in[0,1]:|g(s)|>t\} \leq C Q_{a} f(t)(t>0)$ for some $C>0$. For the definition and detailed discussion of the so-called Kruglov property, see next section.

THEOREM ([Br] $)$. If $X$ has the Kruglov property and $\left\{f_{k}\right\}_{k=1}^{\infty}$ is a sequence of i.i.d.r.v.'s such that $f_{1} \in A_{2}(X), f_{1} \in L_{2}$ and $\mathbb{E} f_{1}=0$, then (1.1) holds.

The proof of this result given in $[\mathrm{Br}]$ is rather indirect and based on fine estimates of infinitely divisible distributions in r.i. spaces. The novelty of our approach here is twofold. Firstly, we observe that sequences $\left\{f_{k}\right\}_{k=1}^{\infty}$ of independent mean zero r.v.'s in a r.i. space $X$ with the Kruglov property behave very similarly to the sequences of their disjoint translates $\left\{\bar{f}_{k}(\cdot):=f_{k}(\cdot-k+1)\right\}_{k=1}^{\infty}$ in some r.i. space $Z_{X}^{2}$ on the semi-axis $(0, \infty)$ [AS3]. More precisely, in this case there exists a constant $C>0$ such that for every sequence of r.v.'s $\left\{f_{k}\right\}_{k=1}^{\infty} \subset X$ as above, we have

$$
C^{-1}\left\|\sum_{k=1}^{n} \bar{f}_{k}\right\|_{Z_{X}^{2}} \leq\left\|\sum_{k=1}^{n} f_{k}\right\|_{X} \leq C\left\|\sum_{k=1}^{n} \bar{f}_{k}\right\|_{Z_{X}^{2}} .
$$

Here, the r.i. space $Z_{X}^{2}$ consists of all measurable functions $f$ on $(0, \infty)$, such that

$$
\|f\|_{Z_{X}^{2}}:=\left\|f^{*} \chi_{[0,1]}\right\|_{X}+\left\|f^{*} \chi_{[1, \infty)}\right\|_{L_{2}[1, \infty)}<\infty,
$$

where $f^{*}(s)$ is the non-increasing rearrangement of $|f(s)|$ (see next section). The connection of the Kruglov property with the estimates similar to (1.3) explained in detail in [AS1, AS2, AS3] allows us to significantly straighten the arguments in the proof of (1.1) and avoid using infinitely divisible distributions. It is fitting to mention that under a somewhat stronger assumption that $X \supset L_{p}$ for $p<\infty$, the inequality (1.3) had been obtained earlier in [JS], where it is also shown that the left hand side inequality in (1.3) holds in every r.i. space $X$.

To see that the theorem above is an easy corollary of the right hand side inequality (1.3), let $\left\{f_{k}\right\}_{k=1}^{\infty}$ be a sequence of i.i.d.r.v.'s such that $f_{1} \in A_{2}(X), f_{1} \in L_{2}$ and $\mathbb{E} f_{1}=0$. A standard argument shows that (1.1) is an immediate corollary of the following implication: $a=\left(a_{k}\right)_{k=1}^{\infty} \in l_{2} \Rightarrow \sum_{k=1}^{\infty} a_{k} f_{k} \in X$. Now, if $\bar{f}_{a}=\sum_{k=1}^{\infty} a_{k} \bar{f}_{k}$, then we obtain from the definition (1.2)

$$
Q_{a} f_{1}(t)=\lambda\left\{s>0:\left|\bar{f}_{a}(s)\right|>t\right\} \quad(t>0) .
$$


Therefore, the assumption $f_{1} \in A_{2}(X)$ implies $f_{a}^{*} \chi_{[0,1]} \in X$ and similarly the assumption $f_{1} \in L_{2}$ guarantees that $\bar{f}_{a} \in L_{2}(0, \infty)$ for every $a=\left(a_{k}\right)_{k=1}^{\infty} \in l_{2}$. At last, the definition of the space $Z_{X}^{2}$ (see (1.4)) yields $\bar{f}_{a} \in Z_{X}^{2}$, and due to (1.3), we conclude $\sum_{k=1}^{\infty} a_{k} f_{k} \in X$.

The second novelty of our approach is related to the study of the class of r.i. spaces $X$ such that (1.1) holds for every sequence of i.i.d.r.v.'s $\left\{f_{k}\right\}_{k=1}^{\infty} \subset X$. To study the question when $f \in A_{2}(X)$ for any given $f \in X$, we employ interpolation methods. An interpolation type assumption on $X$ which we use here is very easy to verify in concrete situations and, in fact, it allows us to completely eliminate from consideration a rather vague condition $f \in A_{2}(X)$.

Our approach allows us to answer in full two open questions from [CD] and $[\mathrm{Br}]$. The negative answer to the question raised in [Br, p. 71] on whether the conditions (a)-(c) are sufficient to guarantee that (1.1) holds is given in Corollary 3.6 below. There, we also answer negatively the question [CD] whether an arbitrary i.i.d. sequence of r.v.'s spans a Hilbertian subspace in the space $L_{2, q}, 0<q<2$. We note that a negative answer to the question of N. L. Carothers and S. J. Dilworth has been announced in $[\mathrm{N}]$, but the proof given there is incomplete.

In view of the necessity of conditions (a)-(c) above, we shall assume below that $X \subseteq L_{2}$ and that the sequence $\left\{f_{k}\right\}_{k=1}^{\infty}$ consists of mean zero r.v.'s. The main result of this article is Theorem 3.1 below.

\section{Definitions and preliminaries}

2.1. Rearrangement invariant spaces. A Banach space $X$ of real-valued Lebesgue measurable functions (with identification $\lambda$-a.e.) on the interval $J$, where $J=[0,1]$ or $[0, \infty)$, will be called rearrangement invariant (r.i.) if

(i) $X$ is an ideal lattice, that is, if $y \in X$, and if $x$ is any measurable function on $J$ with $0 \leq|x| \leq|y|$ then $x \in X$ and $\|x\|_{X} \leq\|y\|_{X}$

(ii) if $y \in X$, and if $x$ is any measurable function on $J$ with $x^{*}=y^{*}$, then $x \in X$ and $\|x\|_{X}=\|y\|_{X}$.

Here, $x^{*}$ denotes the non-increasing, right-continuous rearrangement of $x$ given by

$$
x^{*}(t)=\inf \left\{\tau \geq 0: n_{|x|}(\tau) \leq t\right\}, \quad t>0,
$$

where $n_{|x|}(\tau):=\lambda\{s \geq 0:|x(s)|>\tau\}$.

The Köthe dual $X^{\times}$of an r.i. space $X$ on the interval $J$ consists of all measurable functions $y$ for which

$$
\|y\|_{X^{\times}}:=\sup \left\{\int_{J} x(t) y(t) d t: x \in X,\|x\|_{X} \leq 1\right\}<\infty .
$$

If $X^{*}$ denotes the Banach dual of $X$, it is known that $X^{\times} \subset X^{*}$ and $X^{\times}=X^{*}$ if and only if $X$ is separable. The norm $\|\cdot\|_{X}$ on $X$ is said to be a Fatou norm if the unit ball of $X$ is closed in $E$ with respect to almost everywhere convergence. The norm on the r.i. space $X$ is a Fatou norm if and only if the natural embedding $X \hookrightarrow X^{\times \times}$of $X$ into its Köthe bidual is an isometry. 
An important characteristic of a r.i. space $X$ is the so-called fundamental function $\varphi_{X}(t)=\left\|\mathbf{1}_{(0, t]}\right\|_{X}$, where we denote by $\mathbf{1}_{e}$ the indicator function of a measurable set $e \subset[0, \infty)$.

Every increasing concave function $\varphi$ on $[0,1], \varphi(0)=0$, generates the Lorentz space $\Lambda(\varphi)$ endowed with the norm

$$
\|x\|_{\Lambda(\varphi)}=\int_{0}^{1} x^{*}(t) d \varphi(t) .
$$

It is easy to check that $\varphi_{\Lambda(\varphi)}(t)=\varphi(t)$.

We also recall the definition of the (Lorentz) spaces $L_{p, q}: x \in L_{p, q}$ if and only if the quasi-norm

$$
\|x\|_{p, q}= \begin{cases}\frac{q}{p}\left(\int_{0}^{1}\left(x^{*}(t) t^{1 / p}\right)^{q} \frac{d t}{t}\right)^{1 / q}, & q<\infty, \\ \sup x^{*}(t) t^{1 / p}, & q=\infty,\end{cases}
$$

is finite. $L_{p, q}$-spaces play a significant role in the interpolation theory [KPS], [LT]. The expression $\|\cdot\|_{p, q}$ is a norm if $1 \leq q \leq p$ and is equivalent to a (Banach) norm if $q>p \geq 1$.

Let $X$ be a r.i. space on $[0,1]$. We shall also work with a r.i. space $X(\Omega, \mu)$ of r.v.'s on a probability space $(\Omega, \mu)$ given by

$$
X(\Omega, \mu):=\left\{f \in L_{1}(\Omega, \mu): f^{*} \in X\right\}, \quad\|f\|_{X(\Omega, \mu)}:=\left\|f^{*}\right\|_{X} .
$$

Here, the decreasing rearrangement $f^{*}$ is calculated with respect to the measure $\mu$ on $\Omega$. We denote by $S(\Omega)(=S(\Omega, \mu))$ the linear space of all measurable finite a.e. functions on a given measure space $(\Omega, \mu)$ equipped with the topology of convergence locally in measure.

For basic properties of rearrangement invariant spaces, we refer to the monographs [KPS], [LT].

2.2. Interpolation functors. Throughout this paper, we denote by $\vec{X}=\left(X_{0}, X_{1}\right)$ a (compatible) Banach couple [KPS], [LT], [BK]. The sum $X_{0}+X_{1}$ and the intersection $X_{0} \cap X_{1}$ are equipped with the usual norms:

$$
\begin{gathered}
\|x\|_{X_{0}+X_{1}}=\inf \left\{\left\|x^{0}\right\|_{X_{0}}+\left\|x^{1}\right\|_{X_{1}}: x=x^{0}+x^{1}, x^{0} \in X_{0}, x^{1} \in X_{1}\right\}, \\
\|x\|_{X_{0} \cap X_{1}}=\max \left\{\|x\|_{X_{0}},\|x\|_{X_{1}}\right\} .
\end{gathered}
$$

Let $\vec{X}=\left(X_{0}, X_{1}\right)$ and $X$ be a Banach space such that $X_{0} \cap X_{1} \subseteq X \subseteq X_{0}+X_{1}$. We say that $X$ is an interpolation space between $X_{0}$ and $X_{1}$ if any bounded linear operator $A: X_{0}+X_{1} \rightarrow X_{0}+X_{1}$ which maps $X_{i}$ boundedly into $X_{i}(i=0,1)$ also maps $X$ boundedly into $X$. The set of all interpolation spaces between $X_{0}$ and $X_{1}$ will be denoted by $\operatorname{Int}\left(X_{0}, X_{1}\right)$.

The $K$-functional $K(t, x ; \vec{X})$ is defined for $x \in X_{0}+X_{1}$ and $t>0$ by setting

$$
K(t, x ; \vec{X})=\inf \left\{\left\|x^{0}\right\|_{X_{0}}+t\left\|x^{1}\right\|_{X_{1}}: x=x^{0}+x^{1}, x^{0} \in X_{0}, x^{1} \in X_{1}\right\} .
$$

Let $\Phi$ be a Banach lattice over $\left((0, \infty), \frac{d t}{t}\right)$ satisfying the condition $\min (1, t) \in \Phi$. Denote by $\left(X_{0}, X_{1}\right)_{\Phi}^{K}$ the set of all elements $x \in X_{0}+X_{1}$ such that $K\left(t, x, X_{0}, X_{1}\right) \in \Phi$ endowed with the norm $\|x\|_{\left(X_{0}, X_{1}\right)_{\Phi}^{K}}=\|K(t, x ; \vec{X})\|_{\Phi}$. 
It is well known that the map $\left(X_{0}, X_{1}\right) \mapsto\left(X_{0}, X_{1}\right)_{\Phi}^{K}$ is an interpolation functor (see e.g. [BK, 3.3.12]). The latter means, in particular, that if $\vec{X}=\left(X_{0}, X_{1}\right)$ is a Banach couple, then the space $\left(X_{0}, X_{1}\right)_{\Phi}^{K} \in \operatorname{Int}\left(X_{0}, X_{1}\right)$. This interpolation method is called the $K$-method and the lattice $\Phi$ is called the parameter of the $K$-method.

A couple of Banach spaces $\vec{X}=\left(X_{0}, X_{1}\right)$ is said to be a $K$-monotone couple if there exists a constant $C>0$ such that for any $x, y \in X_{0}+X_{1}$ with $K(t, x ; \vec{X}) \leq K(t, y ; \vec{X})$, $t \in(0, \infty)$, there exists a linear operator $A: X_{0}+X_{1} \rightarrow X_{0}+X_{1}$ such that $x=A y$ and such that $A$ is bounded in $X_{0}$ and $X_{1}$ with $\max _{i=0,1}\|A\|_{X_{i} \rightarrow X_{i}} \leq C$.

2.3. The Kruglov property and the operator $\mathcal{K}$ in r.i. spaces. Let $f$ be a r.v. on $[0,1]$ and let $\mathcal{F}_{f}$ be its distribution function. By $\pi(f)$ we denote any r.v. on $[0,1]$ whose characteristic function is given by

$$
\varphi_{\pi(f)}(t)=\exp \left(\int_{-\infty}^{\infty}\left(e^{i t x}-1\right) d \mathcal{F}_{f}(x)\right),
$$

or, equivalently a r.v. $\sum_{i=1}^{N} f_{i}$, where $f_{i}$ 's are independent copies of $f$ and $N$ is a Poisson random variable with parameter 1 independent of the sequence $\left\{f_{i}\right\}$.

Definition. An r.i. space $X$ is said to have the Kruglov property (we write: $X \in \mathbb{K}$ ) if and only if $f \in X \Leftrightarrow \pi(f) \in X$.

This property has been studied and extensively used by M. Sh. Braverman [Br], who noted, in particular, that only the implication $f \in X \Rightarrow \pi(f) \in X$ is non-trivial, since the implication $\pi(f) \in X \Rightarrow f \in X$ is always satisfied [Br, p. 11]. Note that an r.i. space $X \in \mathbb{K}$ if $X \supseteq L_{p}$ for some $p<\infty$ [Br, Theorem 2, p. 16]. Moreover, Kruglov's theorem [K] gives that exponential Orlicz spaces $L_{N_{p}}$, where $N_{p}(u)$ is equivalent to the function $e^{u^{p}}-1$ for sufficiently large $u>0$, also possess this property if $0<p \leq 1$.

In [AS2] (see also [AS1]) we defined the operator $\mathcal{K}$ on $S([0,1], \lambda)$ which is closely linked with the Kruglov property. From a technical viewpoint, it is more convenient to assume that this operator takes its values in $S(\Omega, \mathcal{P})$, where $(\Omega, \mathcal{P}):=\prod_{k=0}^{\infty}\left([0,1], \lambda_{k}\right)$ (here, $\lambda_{k}$ is Lebesgue measure on $[0,1]$ for every $k \geq 0$ ). Let $\left\{E_{n}\right\}$ be a sequence of pairwise disjoint subsets of $[0,1], m\left(E_{n}\right)=\frac{1}{e \cdot n !}, n \in \mathbb{N}$. For a given $f \in S([0,1], \lambda)$, we set

$$
\mathcal{K} f\left(\omega_{0}, \omega_{1}, \omega_{2}, \ldots\right):=\sum_{n=1}^{\infty} \sum_{k=1}^{n} f\left(\omega_{k}\right) \chi_{E_{n}}\left(\omega_{0}\right)
$$

Let also $\delta:(\Omega, \mathcal{P}) \rightarrow([0,1], \lambda)$ be a measure preserving isomorphism. For every $g \in$ $S(\Omega, \mathcal{P})$, we set $T(g)(x):=g\left(\delta^{-1} x\right), x \in[0,1]$. Note that $T$ is a rearrangement-preserving mapping between $S(\Omega, \mathcal{P})$ and $S([0,1], \lambda)$. So, the distribution function of $T \mathcal{K} f$ is the same as the distribution function of $\mathcal{K} f$. The operator $T \mathcal{K}$ acts on $S([0,1], \lambda)$ and, by an abuse of language, we shall refer to the latter operator as $\mathcal{K}$.

It is important to note that the operator $\mathcal{K}(=T \mathcal{K})$ maps an r.i. space $X$ boundedly into itself if and only if $X$ has the Kruglov property [AS2, Lemma 3.3]. In [AS2], the action of the linear operator $\mathcal{K}$ on various classes of r.i. spaces is studied. In [AS3], we have studied series of independent mean zero r.v.'s in r.i. spaces with the Kruglov property. 
3. Results and proofs. Our main results are the following.

THEOREM 3.1. If $X$ is a r.i. space on $[0,1]$ such that $X \in \operatorname{Int}\left(L_{2}, L_{\infty}\right)$ and either

(i) $\mathcal{K}: X \rightarrow X$, or

(ii) $X$ has Fatou norm and $\mathcal{K}: X \rightarrow X^{\times \times}$,

then there exists $c>0$ such that for any i.i.d. mean zero sequence $\left\{f_{k}\right\}_{k=1}^{\infty} \subset X$ and for every $a=\left(a_{k}\right)_{k=1}^{\infty} \in l_{2}$, the following inequality holds:

$$
\left\|\sum_{k=1}^{\infty} a_{k} f_{k}\right\|_{X} \leq c\left\|f_{1}\right\|_{X}\|a\|_{2}
$$

Proof. For any given $a=\left(a_{k}\right)_{k=1}^{\infty} \in l_{2}$, we define a linear operator $T_{a}: S(0,1) \rightarrow S(0, \infty)$ by setting

$$
T_{a} f(t)=\sum_{k=1}^{\infty} a_{k} f(t-k+1) \mathbf{1}_{(k-1, k]}(t) .
$$

Noting, that for every $f \in L_{2}$ (respectively, $f \in L_{\infty}$ ) we have

$$
\left\|T_{a} f\right\|_{2}=\left(\sum_{k=1}^{\infty} a_{k}^{2} \int_{k-1}^{k} f^{2}(t-k+1) d t\right)^{1 / 2}=\|a\|_{2}\|f\|_{2}
$$

$$
\text { (respectively, }\left\|T_{a} f\right\|_{\infty}=\sup _{k}\left|a_{k}\right|\|f\|_{\infty} \leq\|a\|_{2}\|f\|_{\infty} \text { ) }
$$

we conclude that $T_{a}$ acts boundedly from $L_{2}(0,1)$ into $Z_{L_{2}}^{2}\left(=L_{2}(0, \infty)\right.$ ) (respectively, from $L_{\infty}(0,1)$ into $\left.L_{\infty}(0, \infty)\right)$. Combining the inequality

$$
\|g\|_{Z_{L_{\infty}}^{2}} \leq 2\|g\|_{L_{\infty}(0, \infty) \cap L_{2}(0, \infty)}
$$

(in fact, $Z_{L_{\infty}}^{2}=L_{\infty}(0, \infty) \cap L_{2}(0, \infty)$ ) with (3.2), we obtain:

$$
\left\|T_{a} f\right\|_{Z_{L_{\infty}}^{2}} \leq 2\|a\|_{2}\|f\|_{\infty}, \quad \forall f \in L_{\infty}(0,1),
$$

i.e. $T_{a}$ acts boundedly from $L_{\infty}(0,1)$ into $Z_{L_{\infty}}^{2}$. In order to "interpolate" inequalities $(3.2)$ and (3.3) and extend them to an arbitrary r.i. space $X \in \operatorname{Int}\left(L_{2}, L_{\infty}\right)$, we will need the following auxiliary lemmas, the first of them is proved in [A, Lemma 4].

Lemma 3.2. For any Banach couple $\left(X_{0}, X_{1}\right)$ and an arbitrary parameter $\Phi$ of the $K$-method the following equality holds:

$$
\left(X_{0}, X_{0} \cap X_{1}\right)_{\Phi}^{K}=\left(X_{0}, X_{1}\right)_{\Phi}^{K} \cap X_{0} .
$$

Since the couple $\left(L_{2}, L_{\infty}\right)$ is $K$-monotone [LS] and since $X \in \operatorname{Int}\left(L_{2}, L_{\infty}\right)$, we have by [BK, Theorem 3.3.20] that there exists a parameter $\Phi$ of the $K$-method such that

$$
X=\left(L_{2}, L_{\infty}\right)_{\Phi}^{K}
$$

LEMMA 3.3. If the parameter $\Phi$ is such that (3.4) holds, then

$$
Z_{X}^{2}(0, \infty)=\left(L_{2}(0, \infty), L_{\infty}(0, \infty)\right)_{\Phi}^{K} \cap L_{2}(0, \infty)
$$

up to norm equivalence.

Proof. Set

$$
V:=\left(L_{2}(0, \infty), L_{\infty}(0, \infty)\right)_{\Phi}^{K} \quad \text { and } \quad W:=V \cap L_{2}(0, \infty)
$$


The projection $P f(t)=f \mathbf{1}_{[0,1]}(t), f \in S(0, \infty)$ acts from $L_{p}(0, \infty)$ onto $L_{p}$ with norm 1 , for every $1 \leq p \leq \infty$. Hence, for every $f \in W$, we have $\|f\|_{W} \geq\left\|f^{*} \mathbf{1}_{[0,1]}\right\|_{V}=\left\|f^{*} \mathbf{1}_{[0,1]}\right\|_{X}$. This yields immediately $\|f\|_{W} \geq 2^{-1}\|f\|_{Z_{X}^{2}}$.

In order to prove the converse inequality, we note first that $X \subset L_{2}$ (by assumption) and so for some $c \geq 1$, we have $\|f\|_{2} \leq c\|f\|_{X}, \forall f \in X$. Hence,

$$
\|f\|_{L_{2}(0, \infty)} \leq c\left\|f^{*} \mathbf{1}_{[0,1]}\right\|_{X}+\left\|f^{*} \mathbf{1}_{[1, \infty)}\right\|_{L_{2}} \leq c\|f\|_{Z_{X}^{2}}
$$

and

$$
\begin{aligned}
\|f\|_{V} & \leq\left\|f^{*} \mathbf{1}_{[0,1]}\right\|_{X}+\left\|f^{*} \mathbf{1}_{[1, \infty)}\right\|_{\left(L_{2} \cap L_{\infty}\right)(0, \infty)} \\
& \leq\left\|f^{*} \mathbf{1}_{[0,1]}\right\|_{X}+f^{*}(1)+\left\|f^{*} \mathbf{1}_{[1, \infty)}\right\|_{L_{2}(1, \infty)} \\
& \leq\left(1+\left\|\mathbf{1}_{[0,1]}\right\|_{X}^{-1}\right)\left\|f^{*} \mathbf{1}_{[0,1]}\right\|_{X}+\left\|f^{*} \mathbf{1}_{[1, \infty)}\right\|_{L_{2}(1, \infty)} \\
& \leq\left(1+\varphi_{X}(1)^{-1}\right)\|f\|_{Z_{X}^{2}},
\end{aligned}
$$

where $\varphi_{X}(u)$ is the fundamental function of $X$. Finally, we have

$$
\|f\|_{W} \leq \max \left(c, 1+\varphi_{X}(1)^{-1}\right)\|f\|_{Z_{X}^{2}}
$$

and the lemma is proved.

We continue the proof of Theorem 3.1. Combining (3.2), (3.3) and Lemmas 3.2 and 3.3 , we see that there exists $c_{1}>0$ depending only on the space $X$ such that

$$
\left\|T_{a} f\right\|_{Z_{X}^{2}} \leq c_{1}\|a\|_{2}\|f\|_{X}, \quad \forall f \in X, a \in l_{2} .
$$

On the other hand, the assumptions on the space $X$ made in Theorem 3.1 allow us to use [AS3, Theorem 1]. In particular, for any sequence $\left\{f_{k}\right\}_{k=1}^{n} \subset X$ of i.i.d. mean zero r.v.'s and for any $n \in \mathbb{N}$, we have

$$
\left\|\sum_{k=1}^{n} a_{k} f_{k}\right\|_{X} \leq c_{2}\left\|\sum_{k=1}^{n} a_{k} \bar{f}_{k}\right\|_{Z_{X}^{2}}, \quad \forall a_{1}, a_{2}, \ldots, a_{n} \in \mathbb{R} .
$$

Since the functions $f_{k}, k=1,2 \ldots$ are identically distributed the same holds also for the functions $\sum_{k=1}^{n} a_{k} \bar{f}_{k}$ and $T_{a^{n}} f_{1}$, where $a^{n}=\left(a_{k}^{n}\right), a_{k}^{n}=a_{k}(k \leq n)$ and $a_{k}^{n}=0(k>n)$. So (3.5) and (3.6) yield the following inequality

$$
\left\|\sum_{k=1}^{n} a_{k} f_{k}\right\|_{X} \leq c_{1} c_{2}\left\|f_{1}\right\|_{X}\|a\|_{2}, n=1,2, \ldots
$$

Since the last inequality is equivalent to (3.1), the theorem is proved.

The condition that (1.1) holds for an arbitrary sequence $\left\{f_{k}\right\}_{k=1}^{\infty} \subset X$ of i.i.d. mean zero r.v.'s is formally weaker than the assertion of of Theorem 3.1. Nevertheless, the following result holds.

THEOREM 3.4. Let $X$ be a r.i. space on $[0,1]$. Any sequence $\left\{f_{k}\right\}_{k=1}^{\infty} \subset X$ of i.i.d. mean zero r.v.'s spans a Hilbertian subspace in $X$ if and only if the inequality (3.1) holds for any such sequence and some constant $c>0$, which depends only on $X$.

Proof. We only need to show that if $X$ is a r.i. space such that any sequence $\left\{f_{k}\right\}_{k=1}^{\infty} \subset X$ of i.i.d. mean zero r.v.'s spans a Hilbertian subspace in $X$, then we have the inequality (3.1). Following [N], we define the set $A(X)$ of all sequences $a=\left(a_{k}\right)_{k=1}^{\infty}$ such that the 
series $\sum_{k=1}^{\infty} a_{k} f_{k}$ converges in $X$ for any sequence $\left\{f_{k}\right\}_{k=1}^{\infty} \subset X$ of i.i.d. mean zero r.v.'s. Note that the set $X_{0}:=\left\{f \in X: \int_{0}^{1} f(x) d x=0\right\}$ is a closed subspace in $X$.

For any $a=\left(a_{k}\right)_{k=1}^{\infty} \in A(X)$, we define a linear operator $T_{a}^{n}: X_{0} \rightarrow X(\Omega, \mu)$ by setting

$$
T_{a}^{n} f\left(\omega_{1}, \omega_{2}, \ldots\right)=\sum_{k=1}^{n} a_{k} f\left(\omega_{k}\right), n \in \mathbb{N} .
$$

Here, $\Omega=[0,1]^{\infty}$ and $\mu=\prod_{k=1}^{\infty} \lambda_{k}$. Since $\left\|T_{a}^{n} f\right\|_{X(\Omega, \mu)} \leq \sum_{k=1}^{n}\left|a_{k}\right|\|f\|_{X}$, the operator $T_{a}^{n}$ is bounded for every $n \in \mathbb{N}$. Moreover, by the definition of $A(X)$ we have

$$
\sup _{n}\left\|T_{a}^{n} f\right\|_{X(\Omega, \mu)}<\infty, \forall f \in X_{0} .
$$

Therefore, applying the Banach-Steinhaus principle, we obtain

$$
\sup _{f \in X_{0},\|f\|_{X} \leq 1} \sup _{n \in \mathbb{N}}\left\|\sum_{k=1}^{n} a_{k} f\left(\omega_{k}\right)\right\|_{X_{(\Omega, \mu)}}<\infty
$$

for all $a \in A(X)$, or equivalently

$$
\sup _{\left\{f_{k}\right\}_{k=1}^{\infty},\left\|f_{1}\right\|_{X} \leq 1}\left\|\sum_{k=1}^{\infty} a_{k} f_{k}\right\|_{X}<\infty,
$$

where $\left\{f_{k}\right\}_{k=1}^{n}$ is an arbitrary i.i.d. mean zero sequence of r.v.'s from $X$. Now, if we define $\|a\|_{A(X)}$ to be equal to the supremum in (3.7), then

$$
\left\|\sum_{k=1}^{\infty} a_{k} f_{k}\right\|_{X} \leq\|a\|_{A(X)}\left\|f_{1}\right\|_{X} .
$$

Using standard arguments it is not hard to show that $\left(A(X),\|\cdot\|_{A(X)}\right)$ is a Banach space. Moreover, it is easy to see that convergence in $A(X)$ implies pointwise convergence. Applying the Closed Graph Theorem, we may conclude that the embedding $l_{2} \subseteq A(X)$ given by the assumption on of the theorem is continuous, in other words, $\|a\|_{A(X)} \leq c\|a\|_{2}$ $\left(\forall a \in l_{2}\right)$. Now, inequality (3.1) follows directly from (3.8). The theorem is proved.

COROLlary 3.5. Let $X$ be a r.i. space on [0,1] such that inequality (1.1) holds for any sequence $\left\{f_{k}\right\}_{k=1}^{\infty} \subset X$ of i.i.d. mean zero r.v.'s. Then there exists $c>0$ such that for any $n \in \mathbb{N}$, any sequence of disjointly supported and identically distributed functions $\left\{g_{k}\right\}_{k=1}^{n} \in X,\left\|g_{1}\right\|_{X}=1$ and any $a_{k} \in \mathbb{R}, k=1,2, \ldots, n$ the following inequality holds:

$$
\left\|\sum_{k=1}^{n} a_{k} g_{k}\right\|_{X} \leq c\left(\sum_{k=1}^{n} a_{k}^{2}\right)^{1 / 2} \text {. }
$$

In particular,

$$
\left\|\sum_{k=1}^{n} a_{k} \mathbf{1}_{\left[\frac{k-1}{n}, \frac{k}{n}\right]}\right\|_{X} \leq c \varphi_{X}(1 / n)\left(\sum_{k=1}^{n} a_{k}^{2}\right)^{1 / 2},
$$

where $\varphi_{X}$ is the fundamental function of the r.i. space $X$.

Proof. It is easy to see that there are sets $\left\{g_{k}^{+}\right\}_{k=1}^{n}$ and $\left\{g_{k}^{-}\right\}_{k=1}^{n}$ of identically distributed r.v.'s such that $\left|g_{k}\right|=g_{k}^{+}+g_{k}^{-}, g_{k}^{+} g_{k}^{-}=0$ and $\int_{0}^{1} g_{k}^{+}(x) d x=\int_{0}^{1} g_{k}^{-}(x) d x$ for any $k=$ $1,2, \ldots, n$. Setting $g_{k}^{\prime}=g_{k}^{+}-g_{k}^{-}, k=1,2, \ldots, n$ we obtain an identically distributed 
sequence of mean zero r.v.'s. Let $\left\{f_{k}\right\}_{k=1}^{n}$ be a sequence of independent copies of $g_{k}^{\prime}$. We have $\left\|f_{1}\right\|_{X}=\left\|g_{1}^{\prime}\right\|_{X}=\left\|g_{1}\right\|_{X}=1$, and by Theorem 3.4

$$
\left\|\sum_{k=1}^{n} a_{k} f_{k}\right\|_{X} \leq c\left(\sum_{k=1}^{n} a_{k}^{2}\right)^{1 / 2}, \quad \forall a_{1}, a_{2}, \ldots, a_{n} \in \mathbb{R},
$$

with some constant $c>0$ independent of $\left\{g_{k}\right\}_{k=1}^{n}$. We now note that the proof of the left hand side inequality (3) in by [JS, Theorem 1] does not use the assumption that an embedding $L_{p} \subseteq X$ holds for some $p<\infty$ (see also [Br, Lemma 5, p.14-15]). Therefore, applying this inequality to the sequence $\left\{f_{k}\right\}_{k=1}^{n}$ of i.i.d. mean zero r.v.'s, we have

$$
\left\|\sum_{k=1}^{n} a_{k} f_{k}\right\|_{X} \geq \frac{1}{4}\left\|\sum_{k=1}^{n} a_{k} g_{k}^{\prime}\right\|_{X}=\frac{1}{4}\left\|\sum_{k=1}^{n} a_{k} g_{k}\right\|_{X} .
$$

Inequality (3.9) follows from (3.11) and (3.12), and inequality (3.10) is a consequence of $(3.9)$.

COROLlary 3.6. If a r.i. space $X \varsubsetneqq L_{2}$ and $\varphi_{X}(u)=u^{1 / 2}$, then there exists an i.i.d. mean zero sequence $\left\{f_{k}\right\}_{k=1}^{\infty} \subset X$ spanning a subspace in $X$ which is not isomorphic to $l_{2}$.

Proof. If every i.i.d. mean zero sequence of r.v.'s $\left\{f_{k}\right\}_{k=1}^{\infty} \subset X$ spans a subspace isomorphic to $l_{2}$, then by the preceding corollary, the inequality (3.10) would hold. In this case (3.10) may be equivalently re-written as

$$
\left\|\sum_{k=1}^{n} a_{k} \mathbf{1}_{\left[\frac{k-1}{n}, \frac{k}{n}\right]}\right\|_{X} \leq c\left\|\sum_{k=1}^{n} a_{k} \mathbf{1}_{\left[\frac{k-1}{n}, \frac{k}{n}\right]}\right\|_{L_{2}}
$$

for any $n \in \mathbb{N}$ and any $a_{k} \in \mathbb{R}, k=1,2, \ldots, n$. This immediately yields that $L_{2} \subseteq X$, and invoking the assumption, we conclude that $X=L_{2}$, which is not the case, since by the same assumption $X \neq L_{2}$. The corollary is proved.

REMARK 3.7. In particular, if $X=L_{2, q}, 1 \leq q<2$, then it follows from Corollary 3.6 that there exists an i.i.d. mean zero sequence $\left\{f_{k}\right\}_{k=1}^{\infty} \subset L_{2, q}$ spanning a subspace in $L_{2, q}$ which is not isomorphic to $l_{2}$. This answers in the negative a question from [CD, p. 157]. The same answer was earlier stated in [N]; however, the proof there is incomplete. Similarly, the same example also demonstrates that the conditions (a)-(c) on a r.i. space $X$ stated in the Introduction are not sufficient to guarantee that (1.1) holds for every i.i.d. mean zero sequence $\left\{f_{k}\right\}_{k=1}^{\infty} \subset X$ of r.v.'s. This answers in the negative a question in $[\mathrm{Br}, \mathrm{p} .71]$.

Corollary 3.8. If a r.i. space $X$ has a Fatou norm, $X \varsubsetneqq L_{2}$ and $\varphi_{X}(u)=u^{1 / 2}$, then $X \notin \operatorname{Int}\left(L_{2}, L_{\infty}\right)$. In particular, $L_{2, q} \notin \operatorname{Int}\left(L_{2}, L_{\infty}\right)$ for every $1 \leq q<2$.

Proof. Since $X \supset L_{2,1} \supset L_{r}, r>2$, then by [AS2, Corollary 5.4], the operator $K$ : $X \rightarrow X^{\times \times}$is bounded. Therefore, if $X \in \operatorname{Int}\left(L_{2}, L_{\infty}\right)$ then, by Theorem 3.4, every i.i.d. mean zero sequence $\left\{f_{k}\right\}_{k=1}^{\infty} \subset X$ of r.v.'s would span a Hilbertian subspace in $X$. This contradicts the assertion of Corollary 3.6.

REMARK 3.9. A proof of a similar result to that of the preceding corollary by a different method may be found also in [MM, Theorem 5]. 
COROLlARY 3.10. Let $\varphi$ be an increasing and concave function on $[0,1]$ such that $t^{\frac{1}{2}} \leq$ $C_{1} \cdot \varphi(t), 0<t \leq 1$ and

$$
\sum_{k=1}^{\infty} \varphi\left(\frac{t^{k}}{k !}\right) \leq C_{2} \varphi(t), \quad 0<t<1,
$$

for some constants $C_{1}$ and $C_{2}$. Then every i.i.d. mean zero sequence of r.v.'s from the Lorentz space $\Lambda(\varphi)$ spans in $\Lambda(\varphi)$ a Hilbertian subspace if and only if there exists a constant $C_{3}>0$ such that

$$
\left(\sum_{k=1}^{n}\left(\varphi\left(\frac{k}{n}\right)-\varphi\left(\frac{k-1}{n}\right)\right)^{2}\right)^{1 / 2} \leq C_{3} \varphi\left(\frac{1}{n}\right), \quad n \in \mathbb{N} .
$$

Proof. First, we suppose that every i.i.d. mean zero sequence of r.v.'s from the Lorentz space $\Lambda(\varphi)$ spans in $\Lambda(\varphi)$ a Hilbertian subspace. By the definition of the norm in the Lorentz space $\Lambda(\varphi)$, we have

$$
\left\|\sum_{k=1}^{n} a_{k} \mathbf{1}_{\left[\frac{k-1}{n}, \frac{k}{n}\right]}\right\|_{\Lambda(\varphi)}=\sum_{k=1}^{n} a_{k}^{*}\left(\varphi\left(\frac{k}{n}\right)-\varphi\left(\frac{k-1}{n}\right)\right),
$$

where $\left\{a_{k}^{*}\right\}_{k=1}^{n}$ is the decreasing rearrangement of the sequence $\left\{\left|a_{k}\right|\right\}_{k=1}^{n}$. Thus, it follows from (3.10) that

$$
\sum_{k=1}^{n} a_{k}^{*}\left(\varphi\left(\frac{k}{n}\right)-\varphi\left(\frac{k-1}{n}\right)\right) \leq c \varphi\left(\frac{1}{n}\right)\left(\sum_{k=1}^{n} a_{k}^{2}\right)^{1 / 2}
$$

for any $n \in \mathbb{N}$ and $a_{k} \in \mathbb{R}, k=1,2, \ldots, n$. It is obvious that the last inequality is equivalent to (3.14).

Conversely, suppose that (3.14) holds. Since (3.13) means that the operator $\mathcal{K}$ sends $\Lambda(\varphi)$ into itself [AS2, Theorem 5.1], it follows from the proof of Theorem 3.1 that it is sufficient to verify that for every sequence $a=\left(a_{k}\right)_{k=1}^{\infty} \in l_{2}$, the operator

$$
T_{a} f(t)=\sum_{k=1}^{\infty} a_{k} f(t-k+1) \mathbf{1}_{(k-1, k]}(t)
$$

is bounded from $\Lambda(\varphi)$ into $Z_{\Lambda(\varphi)}^{2}$. Fix $n \in \mathbb{N}$. By the assumption and (3.14), we have

$$
\begin{aligned}
\left\|T_{a} \mathbf{1}_{\left[0, \frac{1}{n}\right]}\right\|_{Z_{\Lambda(\varphi)}^{2}} & =\left\|\sum_{k=1}^{n} a_{k}^{*} \mathbf{1}_{\left(\frac{k-1}{n}, \frac{k}{n}\right]}\right\|_{\Lambda(\varphi)}+\left\|\sum_{k=n+1}^{\infty} a_{k}^{*} \mathbf{1}_{\left(\frac{k-1}{n}, \frac{k}{n}\right]}\right\|_{L_{2}(1, \infty)} \\
& =\sum_{k=1}^{n} a_{k}^{*}\left(\varphi\left(\frac{k}{n}\right)-\varphi\left(\frac{k-1}{n}\right)\right)+\left(\frac{1}{n} \sum_{k=n+1}^{\infty}\left(a_{k}^{*}\right)^{2}\right)^{\frac{1}{2}} \\
& \leq C_{3} \varphi\left(\frac{1}{n}\right)\left(\sum_{k=1}^{n}\left(a_{k}^{*}\right)^{2}\right)^{\frac{1}{2}}+C_{1} \varphi\left(\frac{1}{n}\right)\left(\sum_{k=n+1}^{\infty}\left(a_{k}^{*}\right)^{2}\right)^{\frac{1}{2}} \\
& \leq\left(C_{1}+C_{3}\right) \varphi\left(\frac{1}{n}\right)\|a\|_{l_{2}} .
\end{aligned}
$$

If $h \in(0,1)$, then there exists $n \in \mathbb{N}$ such that $(n+1)^{-1}<h \leq n^{-1}$. Using the argument 
above, we set

$$
\begin{aligned}
\left\|T_{a} \mathbf{1}_{[0, h]}\right\|_{Z_{\Lambda(\varphi)}^{2}} & \leq\left\|T_{a} \mathbf{1}_{\left[0, \frac{1}{n}\right]}\right\|_{Z_{\Lambda(\varphi)}^{2}} \\
& \leq\left(C_{1}+C_{3}\right) \varphi\left(\frac{1}{n}\right)\|a\|_{l_{2}} \leq 2\left(C_{1}+C_{3}\right) \varphi(h)\|a\|_{l_{2}} .
\end{aligned}
$$

Combining this inequality with Corollary 1 to Lemma II.5.2 in [KPS], we see that $T_{a}$ acts boundedly from $\Lambda(\varphi)$ into $Z_{\Lambda(\varphi)}^{2}$ with the norm less or equal to $4\left(C_{1}+C_{3}\right)\|a\|_{l_{2}}$.

\section{References}

[A] S. V. Astashkin, On interpolation of intersections by the real method of interpolation, Algebra i Analiz 17 (2005), 33-69 (in Russian); English transl.: St. Petersburg Math. J. 17 (2006), 239-266.

[AS1] S. V. Astashkin and F. A. Sukochev, Comparison of sums of independent and disjoint functions in symmetric spaces, Math. Notes 76 (2004), 449-454.

[AS2] S. V. Astashkin and F. A. Sukochev, Sums of independent random variables in rearrangement invariant spaces: an operator approach, Israel J. Math. 145 (2005), 125-156.

[AS3] S. V. Astashkin and F. A. Sukochev, Sums of independent functions in symmetric spaces with the Kruglov property, Math. Notes 80 (2006), 630-636.

[BK] Ju. A. Brudnyi and N. Ya. Krugljak, Interpolation Functors and Interpolation Spaces, Vol. I, North-Holland Mathematical Library 47, North-Holland, Amsterdam, 1991.

[Br] M. Sh. Braverman, Independent Random Variables and Rearrangement Invariant Spaces, Cambridge University Press, 1994.

[CD] N. L. Carothers and S. J. Dilworth, Equidistributed random variables in $L_{p, q}$, J. Functional Analysis 84 (1989), 146-159.

[JS] W. B. Johnson and G. Schechtman, Sums of independent random variables in rearrangement invariant function spaces, Ann. Probab. 17 (1989), 789-808.

[K] V. M. Kruglov, A remark on the theory of infinitely divisible laws, Teor. Veroyatnost. i Primenen. 15 (1970), 331-336 (in Russian).

[KPS] S. G. Krein, Ju. I. Petunin and E. M. Semenov, Interpolation of Linear Operators, Translations of Mathematical Monographs 54, Amer. Math. Soc., 1982.

[LT] J. Lindenstrauss and L. Tzafriri, Classical Banach Spaces. II. Function Spaces, Ergeb. Math. Grenzgeb. 97, Springer, Berlin, 1979.

[LS] G. G. Lorentz and T. Shimogaki, Interpolation theorems for the pairs of spaces $\left(L^{p}, L^{\infty}\right)$ and $\left(L^{1}, L^{q}\right)$, Trans. Amer. Math. Soc. 159 (1971), 207-221.

[MM] L. Maligranda and M. Mastyło, Notes on noninterpolation spaces, J. Approx. Theory 56 (1989), 333-347.

[N] S. Ya. Novikov, On the sharpness of inequalities for independent random variables in Lorentz spaces. Izv. Vyssh. Uchebn. Zaved. Mat. 1992, no. 4, 36-38 (in Russian). 
